\title{
Exciton and polarization contributions to optical transition energies in an epitaxial organic monolayer on a dielectric substrate
}

\author{
Mathias Müller, ${ }^{1}$ Eric Le Moal, ${ }^{1, *}$ Reinhard Scholz, ${ }^{2}$ and Moritz Sokolowski ${ }^{1, \dagger}$ \\ ${ }^{1}$ Institut für Physikalische und Theoretische Chemie der Universität Bonn, Wegelerstraße 12, D-53115 Bonn, Germany \\ ${ }^{2}$ Institut für Angewandte Photophysik, Technische Universität Dresden, D-01062 Dresden, Germany
}

(Received 12 May 2011; published 20 June 2011)

\begin{abstract}
Optical emission and excitation spectra of the model molecule perylene-3,4,9,10-tetracarboxylic acid dianhydride adsorbed on an $\mathrm{NaCl}(100)$ surface were measured in the limit of isolated monomers and for two epitaxial monolayer phases $\left(\mathrm{Q}\right.$ and $\left.\mathrm{HB}^{*}\right)$. Intermolecular dipole-dipole coupling and molecular polarization lead to distinct and structure-dependent relative shifts of the exciton energies with respect to the monomer. In combination with calculations of the exciton band structures, this gives insight into the relevant contributions to the transition energies for an interfacial organic film.
\end{abstract}

DOI: 10.1103/PhysRevB.83.241203

PACS number(s): 78.55.Kz, 71.35.Aa, 78.66.Qn

Intermolecular coupling of excited states in condensed phases of semiconducting organic molecules is of major interest with regard to both fundamental and applied aspects. ${ }^{1}$ This is particularly motivated by the development of electronic or optical devices based on semiconducting organic films. To this end, the understanding of delocalization and concomitant energetic shifts of excitations in relation to the structural order of the condensed phase, particularly at interfaces, is important. Well-ordered epitaxial monolayers on surfaces are very attractive in this view since they can be structurally controlled, guarantee a high level of reproducibility, and are technologically relevant. Concerning charged excitations, photoemission ${ }^{2}$ and scanning tunneling spectroscopic ${ }^{3}$ experiments on thin, well-ordered monolayers have been successfully performed. However, for optical excitations, that is, excitons, so far this has been less possible for a variety of reasons. On metal surfaces, strong coupling of states to the metal and quenching of the excitations occurs. ${ }^{4}$ On insulators, often unwanted dewetting and clustering of the molecules prevails, ${ }^{5}$ and structural control by surface-sensitive techniques using electrons is impossible due to charging effects. Hence, the important role of interfacial structures on optical properties so far has not been clarified.

For the probe system considered here, the above experimental difficulties do not arise. We have performed combined structural and optical in situ investigations on diluted molecules and ordered monolayers of the model molecule perylene-3,4,9,10-tetracarboxylic acid dianhydride (PTCDA) on thin epitaxial films of $\mathrm{NaCl}$ on a $\mathrm{Ag}(100)$ surface. These thin $\mathrm{NaCl}$ films $(\sim 30 \AA \text { in thickness })^{6}$ allow the use of surfacesensitive techniques based on electrons, for example, lowenergy electron diffraction (LEED) for structural control. ${ }^{7}$ Due to the high surface quality of $\mathrm{NaCl}$, the PTCDA monolayers are highly ordered and yield optical spectra with very sharp lines. Interestingly, two different monolayer structures of PTCDA can be prepared on $\mathrm{NaCl}(100) .^{7}$ Their optical transitions are both red-shifted, but by different amounts, with respect to the transition of isolated PTCDA molecules on $\mathrm{NaCl}$. From these data, we are able to discriminate the role of the structure-related intermolecular dipole-dipole coupling and the molecular polarization effects on the exciton energies of an interfacial organic layer.

The experiment that comes nearest to the one we report here in terms of the intermolecular coupling is that of Kirstein et al., who studied optical properties of cyanine dye monolayers on a water surface. ${ }^{8}$ However, since Kirstein's experiment was performed at room temperature, the spectral features were much broader than those reported here.

Before we turn to the optical spectra, we briefly review some information on the two ordered structures of PTCDA monolayers on $\mathrm{NaCl}(100){ }^{7}$ There is a quadratic structure, which is commensurate with the underlying $\mathrm{NaCl}$ surface [Fig. 1(a)], first found at room temperature by Burke et al. ${ }^{9}$ on $\mathrm{NaCl}$ bulk crystals. In addition, a metastable, rectangular, incommensurate structure exists [Fig. 1(b)], which is very similar to that of the (102) crystal plane of the bulk phase of PTCDA. It forms upon deposition at temperatures below $\sim 170 \mathrm{~K}$ and transforms into the quadratic structure upon annealing. We refer to these two structures as the $\mathbf{Q}$ and $\mathbf{H B}^{*}$ phases. In both phases, the unit cells contain two PTCDA molecules, adsorbed planar on the surface. From force-field calculations, ${ }^{9}$ there is evidence that Coulomb attractions between the negatively charged $\mathrm{O}$ atoms of the PTCDA and the $\mathrm{Na}^{+}$ions of the $\mathrm{NaCl}$ are important for the favorable wetting of the surface by PTCDA and in particular for the commensurability of the $\mathbf{Q}$ phase.

The transition dipole $\mu$ of the lowest optical fluorescent transition $\left(\mathrm{S}_{0}-\mathrm{S}_{1}\right)$ is oriented along the long axis of the molecule. ${ }^{10}$ In the $\mathbf{Q}$ structure, the nearest neighbor molecules and consequently the related transition dipole moments are exactly perpendicular to each other. In the $\mathbf{H B}^{*}$ structure, due to the rectangular unit cell, the molecular axes include a slightly smaller angle. In our model (see Fig. 1), we assumed that angle to be identical to that previously reported for the (102) plane of the $\beta$ phase of bulk PTCDA, namely $76^{\circ} .{ }^{11}$

Figure 2 (top) displays the fluorescence (FL) and the fluorescence excitation (FLE) spectra of the $\mathbf{Q}$ phase. This and all other spectra were recorded at $20 \mathrm{~K}$. We used excitation powers of 10-300 $\mathrm{mW}$ from an Ar and a dye laser, respectively. The fluorescence light was measured with a spectrograph (focal length $0.3 \mathrm{~m}$ ) equipped with a cooled charge coupled device (CCD) camera. The FL spectrum shows a strongly dominating "zero phonon line" (i.e., 0-0 transition) at $19380 \mathrm{~cm}^{-1}$ with a full width at half-maximum (FWHM) $\gamma_{0-0} \approx 120 \mathrm{~cm}^{-1}$ and well-resolved vibronic modes on the low-energy side. Note that the position of the $0-0$ transition is subject to slight changes in the range of $\pm 20 \mathrm{~cm}^{-1}$ from 


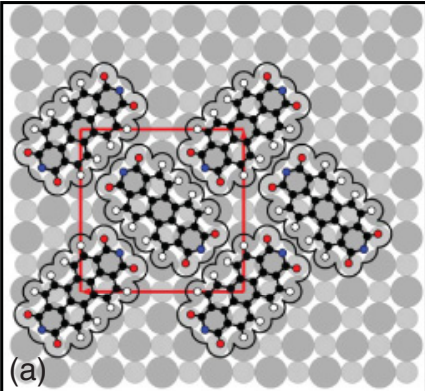

Q phase

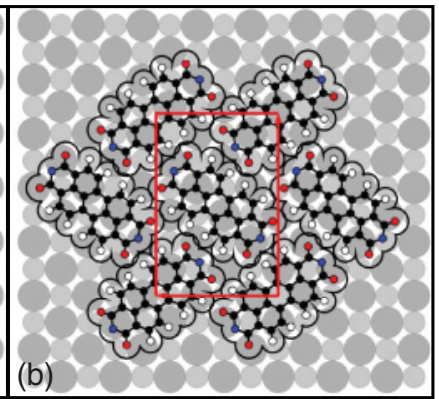

$\mathrm{HB}^{*}$ phase
FIG. 1. (Color online) Models of the two monolayer phases of PTCDA on $\mathrm{NaCl}(100)$ : (a) the commensurate quadratic (Q) structure (unit cell: $16.92 \times 16.92 \AA^{2}$ ) and (b) the incommensurate rectangular herringbone $\left(\mathbf{H B}^{*}\right)$ structure (unit cell: $13.5 \times 20.2 \AA^{2}$ ).

preparation to preparation, most likely due to variations in the size of the two-dimensional (2D) ordered domains of interacting PTCDA molecules. However, the spectrum significantly differs in energy and shape from the spectra of PTCDA bulk crystals, ${ }^{12}$ thin films, ${ }^{13}$ or even double layers on insulators. ${ }^{14}$ These latter spectra are strongly red shifted $\left(\sim 3500 \mathrm{~cm}^{-1}\right)$ with respect to the above monolayer spectrum and exhibit much broader lines due to strong interlayer coupling effects that lead to $\mathrm{H}$-aggregate-like emission properties. ${ }^{14}$ Evidently, this interlayer coupling is absent here. The spectra of PTCDA monolayers on bulk $\mathrm{KCl}(100)$ and $\mathrm{NaCl}(100)$ surfaces were obtained earlier by Schlettwein et al. ${ }^{15}$ and more recently by Dienel et al. ${ }^{16}$ However, the present spectra exhibit much sharper lines and more fine structure; for example, $\gamma_{0-0}$ is smaller by factors of 3 and 7, respectively, mainly due to the lower temperature. Typical decay channels present in PTCDA crystals involving radiative traps or excimer formation ${ }^{12}$ are also absent here. This is corroborated by the FLE spectrum, which mirrors the shape of the FL spectrum and shows only a very small Stokes shift $\left(30 \mathrm{~cm}^{-1}\right)$ due to very little residual disorder. Due to the absence of traps, the shape of the spectra is dominantly determined by the vibrational modes of the individual molecule, as we point out below.

In addition to $2 \mathrm{D}$ ordered layers, a diluted phase of PTCDA (coverage $<1 \%$ of a monolayer) was prepared by deposition at $20 \mathrm{~K}$. In Fig. 2, the FL of this diluted phase (middle) is compared to that of PTCDA in He nanodroplets (bottom). ${ }^{17,18}$ We assume that polarization of the $\mathrm{He}$ is small and that these spectra are close to those of the isolated molecule. On $\mathrm{NaCl}$, the transition is significantly red shifted (by $1307 \mathrm{~cm}^{-1}$, 0-0 at $19680 \mathrm{~cm}^{-1}$ ) due to the stronger polarization of the $\mathrm{NaCl}$ substrate. However, concerning the positions and relative intensities of the vibronic modes, both spectra are in excellent agreement. This reveals that the surface bonding has only a very small influence on the vibrations and hence also on the geometric structure of the molecule. We consider the diluted phase as a reference for single PTCDA molecules (monomers) located on an $\mathrm{NaCl}(100)$ surface.

Comparing the spectra (both FL and FLE) of the monomer phase and that of the $\mathbf{Q}$ phase (Fig. 2), we make the following observations. First, the spectra of the $\mathbf{Q}$ phase are shifted to lower energy by $300 \mathrm{~cm}^{-1}$. A red shift of this order $\left(\sim 240 \mathrm{~cm}^{-1}\right)$ was also detected by Proehl et al. ${ }^{14}$ for

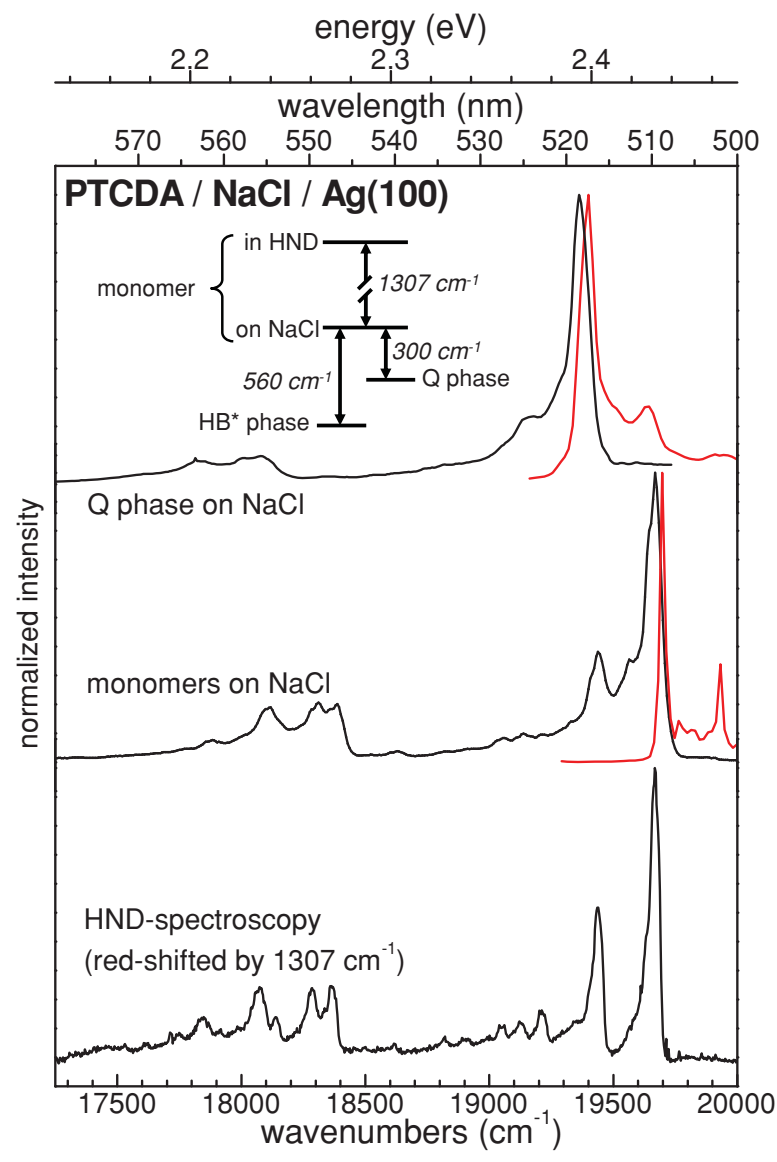

FIG. 2. (Color online) Fluorescence (FL) (left, black) and fluorescence excitation (FLE) spectra (right, red) of the $\mathbf{Q}$ phase (top) and the diluted monomer phase (middle), both measured at a cryostat temperature of $20 \mathrm{~K}$. The excitation wavelengths were $498 \mathrm{~nm}$ and $457 \mathrm{~nm}$, respectively, whereas the detection wavelengths for the FLE spectra were 556 and $543 \mathrm{~nm}$, respectively. In addition, the FL spectrum of single PTCDA molecules in helium nanodroplets (HND) (bottom) ${ }^{17}$ is shown for comparison. This reference spectrum was red shifted by $1307 \mathrm{~cm}^{-1}$ in order to match the positions of the 0-0 transitions with those of the monomer spectra. In the inset, all relevant energy differences of the discussed phases are summarized.

aggregation of PTCDA on mica, but without concomitant structural control. Second, the spectra of the $\mathbf{Q}$ phase exhibit less sharp spectral features compared to those of the diluted phase, likely due to additional soft intermolecular phonon modes. However, the vibrational signatures are the same for both phases and hence the intramolecular vibrational modes are not affected by the 2D packing, demonstrating that the weak coupling situation applies. ${ }^{19}$ Third, the intensity of the zero-phonon line (0-0 transition) with respect to the vibronic side bands of the FL spectrum is increased for the $\mathbf{Q}$ phase (by about a factor of 1.7) with respect to that of the diluted phase. This results from the intermolecular coupling that enhances the FL on the 0-0 line due to superradiant emission. ${ }^{20}$

We now turn to the evolution of the FL when the structure undergoes the phase transition from the $\mathbf{H B}^{*}$ to the $\mathbf{Q}$ phase. Figure 3 displays a series of spectra which were taken for a disordered layer during stepwise annealing. It demonstrates how the $0-0$ transition of the $\mathbf{H B}^{*}$ phase is continuously 


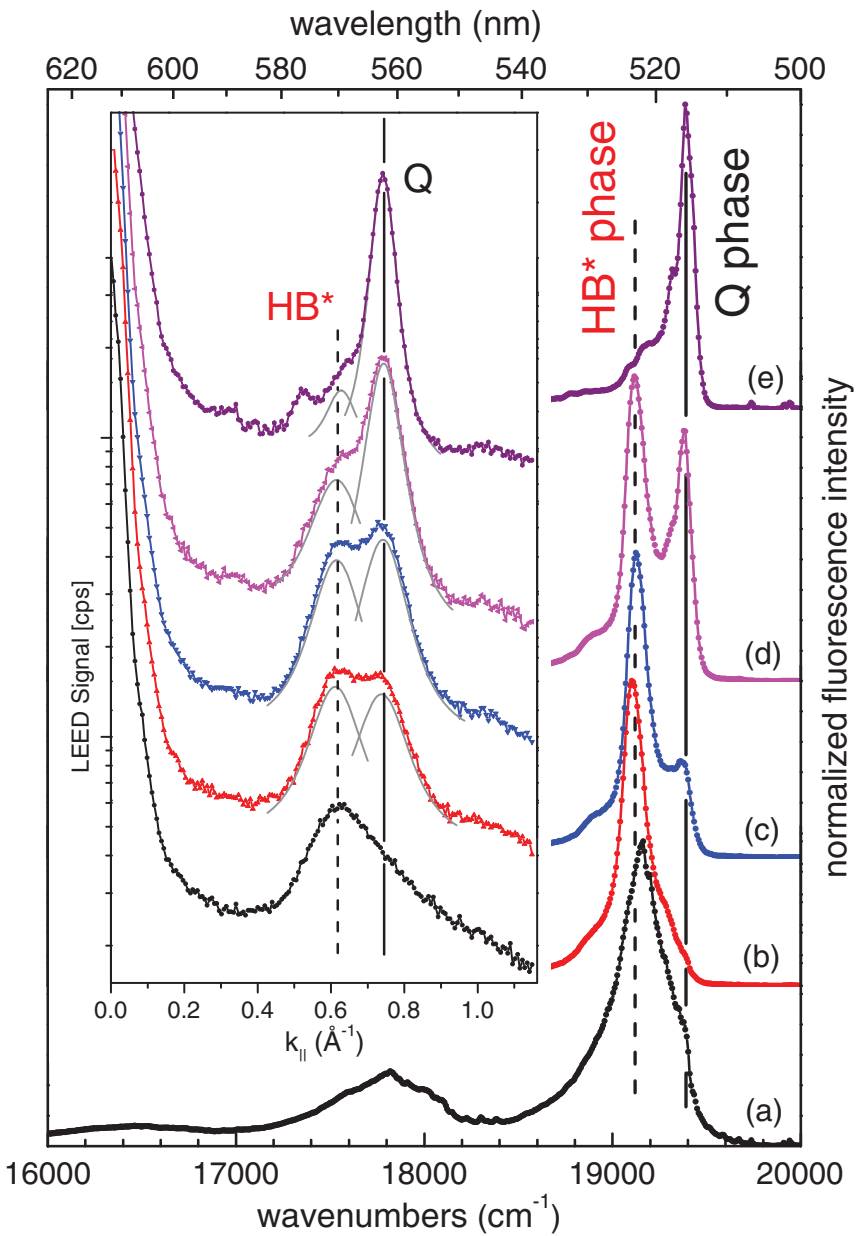

FIG. 3. (Color online) Fluorescence spectra (right) and LEED scans (left) obtained during the transition from the metastable $\mathbf{H B}^{*}$ phase to the $\mathbf{Q}$ phase of PTCDA on $\mathrm{NaCl}(100)$. From (a) to (e), the fraction of the $\mathbf{H B}^{*}$ phase decreases at the expense of the $\mathbf{Q}$ phase due to stepwise thermal annealing of the sample. This can be seen from the LEED scans, where the contributions of the $\mathbf{H B}^{*}$ and the $\mathbf{Q}$ phase are indicated. For further details of the phase transition, see Ref. 7.

replaced by that of the $\mathbf{Q}$ phase, which is blue shifted by $\Delta E_{\mathrm{Q} / \mathrm{HB} *}=260 \mathrm{~cm}^{-1}$ with respect to the $\mathbf{H B}^{*}$ phase $\left(\mathrm{HB}^{*}\right.$ : $0-0$ at $19120 \mathrm{~cm}^{-1}$ ). The inset shows highly resolved LEED scans taken in parallel, which show the relative contributions of the two phases. ${ }^{7}$ From bottom to top, the amount of the metastable $\mathbf{H B}^{*}$ phase decreases and the amount of the stable $\mathbf{Q}$ phase increases until the phase transition is completed. Again, the vibronic side bands of the $\mathbf{H B}^{*}$ phase and $\mathbf{Q}$ phase are identical within the experimental resolution, although this is slightly smaller for the metastable $\mathbf{H B}^{*}$ phase, likely due to some disorder. ${ }^{7}$

The relevant energy differences of the transition energies in all phases are illustrated in the inset in Fig. 2. For further analysis, we performed a calculation of the exciton bands on the basis of the above structures. Band structures of Frenkeltype excitons in organic molecular crystals are commonly calculated in the tight binding approximation..$^{19}$ As a result, the $\boldsymbol{k}$-dependent exciton energies are obtained as

$$
E(\mathbf{k})=E_{0}+D+\sum_{i} M_{i} \exp \left(i \mathbf{k r}_{i}\right) .
$$

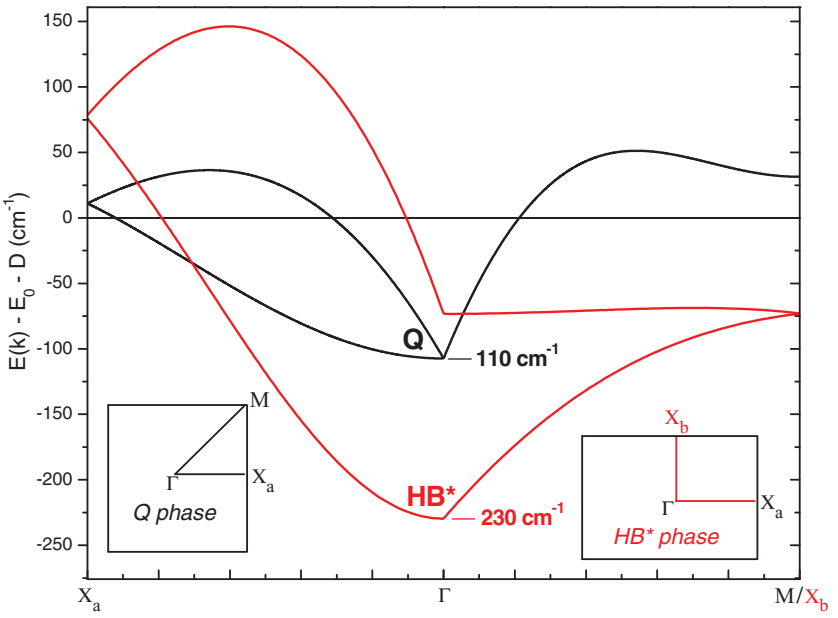

FIG. 4. (Color online) Calculated exciton band structures of the $\mathbf{Q}$ and $\mathbf{H B}^{*}$ phases of PTCDA and corresponding surface Brillouin zones. For further details, see the text.

Here, $M_{i}$ denotes the transition matrix element between two interacting molecules at the origin and displaced by the vector $\mathbf{r}_{i}$, respectively. $E_{0}$ is the transition energy of the isolated molecule, and $D$ describes the polarization effects related to the molecular neighborhood. Quite often $M_{i}$ can be successfully approximated by the interaction of point dipoles of the value of the transition dipole $\mu .{ }^{19}$ For a PTCDA monolayer, this approximation appears reasonable, since the spatial extension of the transition densities is small compared to intermolecular distances. ${ }^{10,21}$ In this case, $M_{i}$ scales with $\chi^{2} \mu^{2}$, whereby $\chi^{2}$ denotes the Franck-Condon factor of the 0-0 transition. ${ }^{19}$ We computed $E(\mathbf{k})-E_{0}-D$ according to Eq. (1) for free-standing PTCDA layers of the $\mathbf{Q}$ and $\mathbf{H B}^{*}$ phases. We used the value $\mu=7.4 \mathrm{D}$, given by Hoffmann et al.,22 and the value $\chi^{2}=0.42$, estimated from spectra of PTCDA in solution. ${ }^{10}$

The calculated exciton bands are displayed in Fig. 4. In the calculation, we surrounded the molecule at the origin by a lattice of $400 \times 400$ unit cells, which gave good convergence of the energies in the range of $1 \%$. Since there are two molecules in both unit cells, two bands for each structure are obtained. However, in the $\Gamma M$ direction, the two bands are degenerate for the $\mathbf{Q}$ phase due to the symmetry of the unit cell. For both structures, a global minimum of energy is found at the center of the Brillouin zone ( $\Gamma$ point). Excitons migrate to this minimum, where they radiatively decay with high probability, since no further vibronic excitations in the final state are required for conservation of momentum as in PTCDA bulk. ${ }^{21}$ As a consequence, the 0-0 line is expected to be the dominant feature in the FL spectra of both phases, in good agreement with the experimental data (Fig. 2).

Molecular ensembles for which this situation applies are referred to as $J$ aggregates. For the $\mathbf{Q}$ phase, this can be understood without a calculation: This phase can be built up as a superposition of two "brickwall" sublattices with perpendicular transition dipoles. The brickstone lattice is known to yield $J$ aggregates. ${ }^{23}$ Due to the perpendicular orientation of the transition dipoles, the dipolar coupling between the two sublattices is weak here and occurs only between 
fourth-nearest-neighbor molecules. As a consequence, conclusions from the simple brickwall lattice remain valid here. For the $\mathbf{H B}^{*}$ phase, the overall bandwidth is larger and the energy at the $\Gamma$ point is lower by about a factor of 2 . This can be intuitively understood from a more effective dipole-dipole coupling due to the generally closer packing and a nonzero coupling of the two molecules in the unit cell due to their nonperpendicular orientation (see Fig. 1).

According to the calculated band structures, the energy minima of the $\mathbf{H B}^{*}$ and $\mathbf{Q}$ phases at the $\Gamma$ point differ by $120 \mathrm{~cm}^{-1}$. This value is significantly smaller than the experimental value $\left(\Delta E_{\mathrm{Q} / \mathrm{HB} *}=260 \mathrm{~cm}^{-1}\right)$. This discrepancy is due to the polarization effects [entering into $D$ in Eq. (1)] that are not taken into account in the calculation of the excitonic dispersion branches. The polarization screening is significantly more effective for the $\mathbf{H B}^{*}$ phase than for the $\mathbf{Q}$ phase, because of the smaller intermolecular distances (cf. Fig. 1). From our experiment, we estimate this contribution to $\Delta E_{\mathrm{Q} / \mathrm{HB}^{*}}$ to be $140 \mathrm{~cm}^{-1}$. The important role of the polarization of the molecular neighborhood is also supported by the observed red shifts of the 0-0 transitions of the $\mathbf{Q}$ phase and $\mathbf{H B}^{*}$ phase with respect to the $0-0$ transition of the PTCDA monomer phase $\left(300 \mathrm{~cm}^{-1}\right.$ and $560 \mathrm{~cm}^{-1}$, respectively). These shifts are underestimated by $190 \mathrm{~cm}^{-1}$ and $330 \mathrm{~cm}^{-1}$ respectively by the calculation (cf. Fig. 4). This difference must also be a consequence of the polarization of the molecular neighborhood, which is present in the condensed monolayers but absent for the monomer phase. ${ }^{19}$ Notably, comparable red shifts in the transition energies previously have been estimated from a time-dependent density functional calculation performed on PTCDA dimers. ${ }^{24}$ This indicates that the major contribution to the polarization energy results from the neighboring molecules.

In summary, we find three effects which determine the optical transition energies of a PTCDA layer on $\mathrm{NaCl}(100)$. The largest contribution is the polarization of the $\mathrm{NaCl}$ substrate, yielding a red shift of $1307 \mathrm{~cm}^{-1}$ with respect to monomers in liquid He. The second contribution stems from the polarization of the molecular environment, yielding shifts of 190 and $330 \mathrm{~cm}^{-1}$ for the two phases $\left(\mathbf{Q} / \mathbf{H B}^{*}\right)$. Finally, the formation of the exciton band structures yields additional red shifts of 110 and $230 \mathrm{~cm}^{-1}$, respectively. The difference of this third contribution for the two phases can be understood from geometric arguments concerning the interaction of transition dipoles. As a result of the latter two contributions, the optical transitions of the two monolayer phases of PTCDA on $\mathrm{NaCl}$ differ by $260 \mathrm{~cm}^{-1}$. We expect similar effects also for other molecules on other dielectric substrates.

Our results hence give an understanding of the role of the structural packing on the optical transition energies of molecules on a dielectric surface. In combination with the calculation of the exciton band structure, our experimental approach allows us to identify the different underlying physical mechanisms and to quantify the energy contributions, while distinguishing those due to the polarization of the molecular layer and those due to the intermolecular (dipole-dipole) coupling for a $2 \mathrm{D}$ layer.

We thank F. Stienkemeier for making unpublished results available. The project was supported by the DFG Research Unit 557 and the Alexander von Humboldt Foundation (E.L.M.).
*Present address: Institut Fresnel, Campus Saint-Jérôme, F-13013

Marseille, France.

${ }^{\dagger}$ sokolowski@pc.uni-bonn.de

${ }^{1}$ H. Klauk (ed.), Organic Electronics (Wiley-VCH, Weinheim, 2006).

${ }^{2}$ H. Kakuta et al., Phys. Rev. Lett. 98, 247601 (2007).

${ }^{3}$ R. Temirov et al., Nature (London) 444, 350 (2006).

${ }^{4}$ W. Gebauer et al., Phys. Rev. B 69, 155431 (2004).

${ }^{5}$ G. Witte and C. Wöll, J. Mater. Res. 19, 1889 (2004).

${ }^{6}$ E. Le Moal et al., Surf. Sci. 603, 2434 (2009).

${ }^{7}$ E. Le Moal et al., Phys. Rev. B 82, 045301 (2010).

${ }^{8}$ S. Kirstein and H. Möhwald, Adv. Mater. 7, 460 (1995).

${ }^{9}$ S. A. Burke et al., Phys. Rev. Lett. 100, 186104 (2008).

${ }^{10}$ E. Engel et al., Phys. Rev. B 73, 245216 (2006).

${ }^{11}$ T. Ogawa et al., Acta Cryst. B 55, 123 (1999).
${ }^{12}$ A. Y. Kobitski et al., Phys. Rev. B 68, 155201 (2003).

${ }^{13}$ V. Bulovic et al., Chem. Phys. 210, 1 (1996).

${ }^{14}$ H. Proehl et al., Phys. Rev. Lett. 93, 097403 (2004).

${ }^{15}$ D. Schlettwein et al., Chem. Mater. 10, 601 (1998).

${ }^{16}$ T. Dienel et al., Adv. Mater. 20, 959 (2008).

${ }^{17}$ M. Dvorak and F. Stienkemeier (unpublished).

${ }^{18}$ M. Wewer and F. Stienkemeier, J. Chem. Phys. 120, 1239 (2004).

${ }^{19}$ D. P. Craig and S. H. Walmsley, Excitons in Molecular Crystals (Benjamin, New York, 1968).

${ }^{20}$ F. C. Spano and S. Mukamel, J. Chem. Phys. 91, 683 (1989).

${ }^{21}$ I. Vragovic and R. Scholz, Phys. Rev. B 68, 155202 (2003).

${ }^{22}$ M. Hoffmann et al., Chem. Phys. 258, 73 (2000).

${ }^{23}$ V. Czikkely et al., Chem. Phys. Lett. 6, 11 (1970).

${ }^{24}$ R. Scholz et al., Phys. Rev. B 72, 245208 (2005). 